\title{
Whole miRNome profiling of the effects of non-digestible carbohydrates on microRNA expression in the healthy human colorectal epithelium: a randomised controlled trial
}

\author{
F.C. Malcomson ${ }^{1}$, N.D. Willis ${ }^{1}$, I. McCallum ${ }^{1}$, L. Xie ${ }^{1}$, B. Lagerwaard ${ }^{1}$, S. Kelly ${ }^{1}$, \\ M. Bradburn ${ }^{1}$, N.J. Belshaw ${ }^{2}$, I.T. Johnson ${ }^{2}$ and J.C. Mathers ${ }^{1}$ \\ ${ }^{1}$ Human Nutrition Research Centre, MRC Centre for Ageing and Vitality, Institute of Cellular Medicine, Newcastle \\ University, Newcastle upon Tyne NE4 5PL and \\ ${ }^{2}$ Institute of Food Research, Norwich Research Park, Norfolk NR4 7UA
}

Colorectal cancer (CRC) risk is strongly modulated by diet and there is convincing evidence that higher intakes of non-digestible carbohydrates (NDCs) reduce CRC risk. Aberrant expression of microRNAs (small, non-coding RNA that regulate gene expression and, consequently, processes such as cell proliferation) is observed in the colorectal tissue, blood, stool and urine from CRC patients ${ }^{(1)}$. Resistant starch (RS), a NDC, and its fermentation product, butyrate, positively modulate the expression of oncogenic microRNAs ${ }^{(2)}$, suggesting that this could be a mechanism through which NDCs protect against CRC.

This study aimed to investigate the effects of supplementation with RS and polydextrose (PD) (two NDCs) for seven weeks on microRNA expression in macroscopically-normal rectal mucosal biopsies from healthy participants taking part in the DISC Study (a randomised, double-blind, placebo-controlled dietary intervention) ${ }^{(3)}$. We used a whole miRNome PCR array to screen 1,008 microRNAs in pooled post-intervention samples from participants allocated to the placebo group and those supplemented with RS and PD.

A total of 111 miRNAs were up- or down-regulated by at least two-fold in the RS + PD group compared with the placebo group. From these, $m i R-26 a, m i R-32$, mir-127, $m i R-133 b, m i R-135, m i R-517, m i R-640$ and $m i R-1287$ were selected for validation in individual samples by qPCR. Post-intervention $m i R-32$ expression was three-fold greater in participants supplemented with RS + PD compared with placebo (Fig. $1 ; P=0.033$ ).

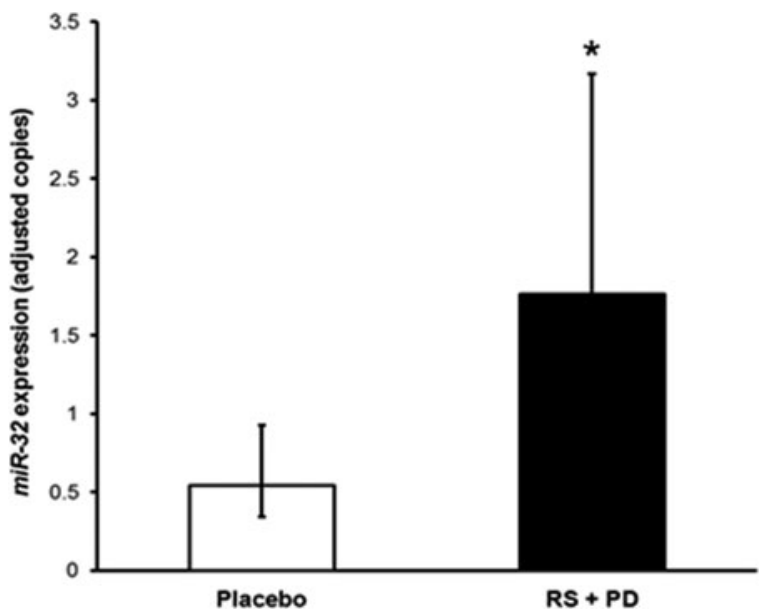

Fig. 1. Post-intervention $m i R-32$ expression in participants supplemented with Placebo and RS + PD.

$m i R-32$ regulates cell proliferation which is dysregulated in CRC. The observed increase in $m i R-32$ with RS + PD could be one of the mechanisms through which these NDCs stimulate cell proliferation in healthy colorectal cells ${ }^{(4)}$. In contrast, NDCs and butyrate (the main energy source for colonocytes) reduce proliferation of CRC cells. Further investigations into the role of microRNAs in mediating this phenomenon in both healthy and cancer tissues and to explore the utility of $m i R-32$ as a diet-responsive biomarker of gut health are warranted.

This study was funded by the BBSRC (BB/H005013/1). Ethical approval for the study was granted on $10^{\text {th }}$ December 2009 (REC No. 09/H0907/77).

1. Schee K, Fodstad O \& Flatmark K (2010) Am J Pathol 177, 1592-1599.

2. Humphreys KJ, Conlon MA, Young GP et al. (2014) Cancer Prev Res 7, 786-795.

3. Malcomson FC, Willis ND, McCallum I et al. (2017) Am J Clin Nutr 105, 400-410.

4. Donohoe DR, Collins LB, Wali A et al. (2012) Mol Cell 48, 612-626. 